\title{
Multi-beam Electron Microscopy: Principles and Applications
}

\author{
Anna Lena Eberle ${ }^{1}$ and Dirk Zeidler ${ }^{1}$ \\ 1. Carl Zeiss Microscopy GmbH, Carl-Zeiss-Str. 22, Oberkochen, Germany
}

Since their invention, scanning electron microscopes (SEMs) have been advanced in a number of aspects, such as increasing their resolution, especially at low electron landing energies, improving their usability through digitization and miniaturization, adding detection schemes such as energy- or wavelength-dispersive X-ray spectrometry (EDX, WDX) and correlative microscopy. The data acquisition rates of SEMs, though, while maintaining both nanometer resolution and high signal-to-noise ratio (SNR), has not seen huge improvements.

The maximum achievable scan speed of any conventional SEM is ultimately limited by the electron dose per pixel required to generate a desired minimal SNR at a given spot size. Two fundamental effects limit the minimum pixel dwell time of single-beam SEMs: (i) reducing dwell time per pixel while retaining SNR requires increasing the beam current, which leads to increased Coulomb interactions between the electrons, thereby blurring the electron beam, and (ii) efficient detectors for secondary electrons in an SEM cannot be operated faithfully at arbitrarily high rates.

Multiple beam SEMs circumvent these two restrictions, as they (i) distribute the charge over a large volume, thus reducing Coulomb effects, and (ii) use one detector for each beam, yielding a much higher total detector bandwidth at relatively low detector rates per beam. A number of different setups for multiple beam SEMs are conceivable, out of which several have been demonstrated in the past, e.g.[1]. We have chosen a single column, multiple beam approach as it combines well-known technology with superb scalability properties.

The details of the setup of this multiple beam SEM have been described elsewhere [2]. Figure 1 summarizes the basic principle of operation: Multiple primary electron beams (depicted blue in the left image) are scanned in a single column in parallel over a sample (right image); one detector per secondary electron beam (green in the left image) enables parallel detection of all beams.

We will give a synopsis on multiple beam scanning electron microscopy approaches, demonstrate the single column, multiple beam technology and its scalability, and present an overview of its latest application results. [3] 


\section{References:}

[1] A. Mohammadi-Gheidari, P. Kruit, Nucl. Instrum. Methods. 645, 60 (2011)

[2] A. L. Eberle et al., J. Microsc. 259, 114 (2015)

[3] The authors thank B. Thiel, M. Malloy, S. Halder, P. Leray, C. Nakakura, J. Michaels, and R. Schalek for insightful discussions.
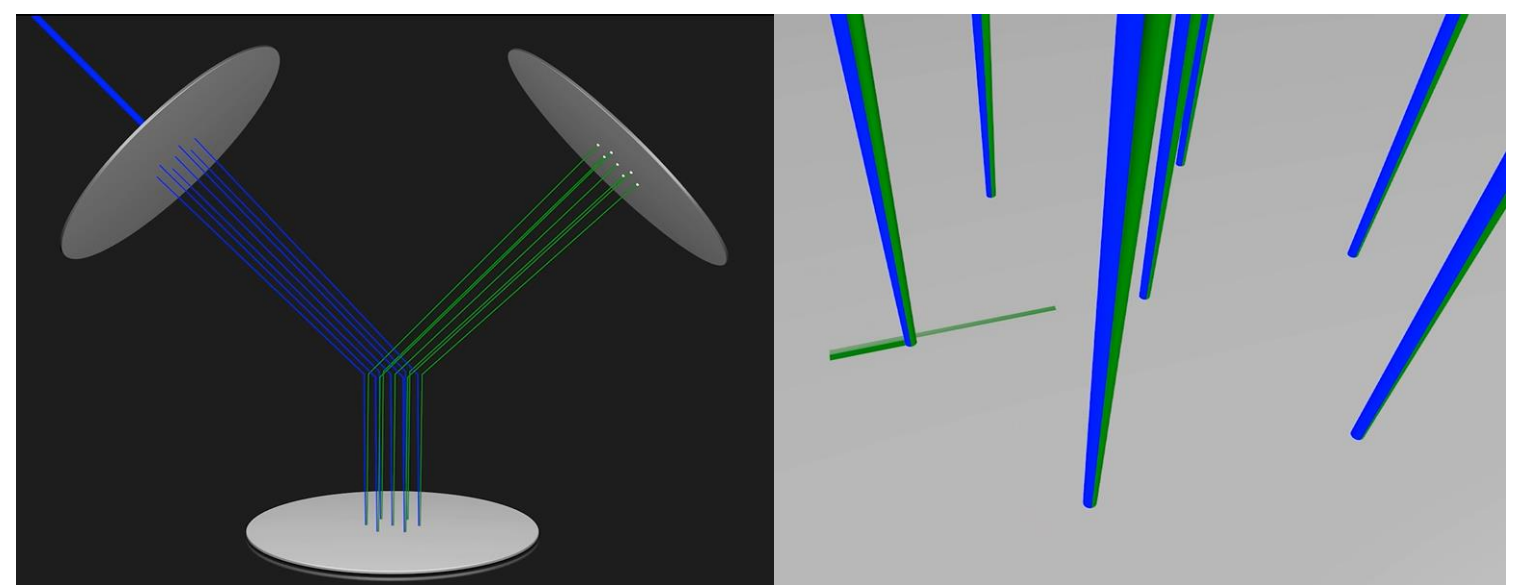

Figure 1. Schematics of the multiple beam SEM. Right: detail of the parallel scanning process. For simplicity, only 7 beams are shown in this sketch. 